\title{
Variance Estimation of Change in Poverty Rates: an Application to the Turkish EU-SILC Survey
}

\author{
Melike Oguz Alper ${ }^{1}$ and Yves G. Berger ${ }^{1}$
}

\begin{abstract}
Interpreting changes between point estimates at different waves may be misleading if we do not take the sampling variation into account. It is therefore necessary to estimate the standard error of these changes in order to judge whether or not the observed changes are statistically significant. This involves the estimation of temporal correlations between cross-sectional estimates, because correlations play an important role in estimating the variance of a change in the cross-sectional estimates. Standard estimators for correlations cannot be used because of the rotation used in most panel surveys, such as the European Union Statistics on Income and Living Conditions (EU-SILC) surveys. Furthermore, as poverty indicators are complex functions of the data, they require special treatment when estimating their variance. For example, poverty rates depend on poverty thresholds which are estimated from medians. We propose using a multivariate linear regression approach to estimate correlations by taking into account the variability of the poverty threshold. We apply the approach proposed to the Turkish EU-SILC survey data.
\end{abstract}

Key words: Linearisation; multivariate regression; stratification; unequal inclusion probabilities.

\section{Introduction}

In order to monitor progress towards agreed policy goals, particularly in the context of the Europe 2020 strategy, there is an interest in evaluating the evolution of social indicators. In order to interpret changes between indicators at different waves, it is important to estimate the standard error of these changes, so that we can judge whether or not observed changes are statistically significant. The poverty rate is an important policy indicator, especially within the context of the Europe 2020 strategy. This rate is defined as the proportion of people with an equivalised total net income below 60 percent of the national median income (Eurostat 2003, 2). This indicator is calculated from the European Union Statistics on Income and Living Conditions (EU-SILC) surveys (Eurostat 2012) which collect yearly information on income, poverty, social exclusion and living conditions from approximately 300,000 households across Europe. The poverty rate is a complex statistic, unlike population totals or means, since it is based on a poverty threshold computed from the median of the income distribution. Hence, there exist two

${ }^{1}$ University of Southampton, University Road Bldg. 58, Southampton SO17 1BJ, UK, Emails: M.OguzAlper@soton.ac.uk and Y.G.Berger@soton.ac.uk

Acknowledgments: Melike Oguz Alper was funded by the Jean Monnet Scholarship Programme, the European Union, and the Economic and Social Research Council (ESRC), United Kingdom. The authors wish to thank to Turkish Statistical Institute (TurkStat) for providing the datasets used in this article. This work was supported by consulting work for the Net-SILC2 project (Atkinson and Marlier 2010). 
sources of variability: one is due to the estimated threshold and the other one comes from the estimated proportion given the estimated threshold (e.g., Berger and Skinner 2003; Verma and Betti 2011).

Several methods to estimate the variance of the poverty rate like resampling and linearisation techniques have been discussed in the literature (e.g., Preston 1995; Deville 1999; Berger and Skinner 2003; Demnati and Rao 2004; Verma and Betti 2005; Osier 2009; Goedemé 2010; Verma and Betti 2011; Muennich and Zins 2011; Osier et al. 2013; Berger and Priam 2015). However, variance of change for the poverty rate has been studied in only limited number of papers (e.g., Betti and Gagliardi 2007; Muennich and Zins 2011; Osier et al. 2013; Berger and Priam 2015). Berger and Priam (2010, 2015) proposed an estimator for the variance of change which takes into account the complexities of the sampling design, such as stratification, unequal probabilities, clustering and rotation (see also Osier et al. 2013). The approach proposed relies neither on the second-order inclusion probabilities nor on the resampling methods, unlike its competitors (Betti and Gagliardi 2007; Wood 2008; Muennich and Zins 2011, 20). It is based on a multivariate linear regression (general linear model) approach that can be easily implemented by any statistical software (Berger and Priam 2015). Berger et al. (2013) show how it can be implemented in SPSS.

The estimator proposed by Berger and Priam (2010, 2015) ignores the sampling variability due to the poverty threshold by treating the poverty rate as a ratio. In Sections 4 and 5, we show how this approach can be adjusted to take into account the sampling variability of the poverty threshold. In Section 6, we compare the approach proposed with the more simple approach proposed by Berger and Priam (2010, 2015; see also Osier et al. 2013) via a series of simulations. In Section 7, we apply the approach proposed to the Turkish EU-SILC survey data. The variance estimator proposed depends on a bandwidth used for the estimation of the density. We also show how sensitive the variance estimates are to the chosen bandwidth parameter by considering different bandwidth parameters.

\section{Rotating Sampling Designs}

With rotating panel surveys, it is common practice to select new units in order to replace old units that have been in the survey for a specified number of waves (e.g., Gambino and Silva 2009; Kalton 2009). The units sampled on both waves usually represent a large fraction of the first-wave sample. This fraction is called the fraction of the common sample. For example, for the EU-SILC surveys, this fraction is 75 percent. For the Canadian labour force survey and the British labour force survey, this fraction is 80 percent. For the Finnish labour force survey, this fraction is 60 percent. We consider that the sample design is such that the common sample has a fixed number of units. Throughout this article, we assume that the sampling fractions are negligible, that is, $\left(1-\pi_{t ; i}\right) \approx 1$, where $\pi_{t ; i}$ denote the inclusion probabilities of unit $i$ at wave $t$.

\section{Estimation of Change of a Poverty Rate}

Let $s_{1}$ and $s_{2}$ be the samples selected at Wave 1 and Wave 2 respectively. Suppose, we wish to estimate the absolute net change $\Delta=\theta_{2}-\theta_{1}$ between two population 
poverty rates $\theta_{1}$ and $\theta_{2}$, from Wave 1 and Wave 2 respectively. Suppose that $\Delta$ is estimated by $\hat{\Delta}=\hat{\theta}_{2}-\hat{\theta}_{1}$; where $\hat{\theta}_{1}$ and $\hat{\theta}_{2}$ are the cross-sectional estimators of poverty rates defined by

$$
\begin{gathered}
\hat{\theta}_{1}=\frac{\hat{\tau}_{1}}{\hat{\tau}_{2}}=\frac{\sum_{i \in s_{1}} \delta\left\{y_{1 ; i} \leq 0.6 \hat{Y}_{1 ; 0.5}\right\} \pi_{1 ; i}^{-1}}{\sum_{i \in s_{1}} \pi_{1 ; i}^{-1}} \text { and } \\
\hat{\theta}_{2}=\frac{\hat{\tau}_{3}}{\hat{\tau}_{4}}=\frac{\sum_{i \in s_{2}} \delta\left\{y_{2 ; i} \leq 0.6 \hat{Y}_{2 ; 0.5}\right\} \pi_{2 ; i}^{-1}}{\sum_{i \in s_{2}} \pi_{2 ; i}^{-1}},
\end{gathered}
$$

where $y_{t ; i}$ is the net equivalised income (see Eurostat 2003,2) of individual $i$ at wave $t$ and $\hat{Y}_{t ; 0.5}$ is the estimate of the median of the population income distribution at wave $t(t=1,2)$. The function $\delta\{A\}=1$ when $A$ is true, and $\delta\{A\}=0$ otherwise.

The design-based variance of the estimator of change $\hat{\Delta}$ is given by

$$
\operatorname{var}(\hat{\Delta})=\operatorname{var}\left(\hat{\theta}_{1}\right)+\operatorname{var}\left(\hat{\theta}_{2}\right)-2 \operatorname{corr}\left(\hat{\theta}_{1}, \hat{\theta}_{2}\right) \sqrt{\operatorname{var}\left(\hat{\theta}_{1}\right) \operatorname{var}\left(\hat{\theta}_{2}\right)} \cdot
$$

Standard design-based estimators can be used to estimate the cross-sectional variances $\operatorname{var}\left(\hat{\theta}_{1}\right)$ and $\operatorname{var}\left(\hat{\theta}_{2}\right)$ (e.g., Deville 1999). The correlation corr $\left(\hat{\theta}_{1}, \hat{\theta}_{2}\right)$ is the most difficult part to estimate as $\hat{\theta}_{1}$ and $\hat{\theta}_{2}$ are estimated from different samples because of the rotation. Estimation of the covariance term has been discussed in several papers (Kish 1965, 457-458; Tam 1984; Laniel 1987; Nordberg 2000; Holmes and Skinner 2000; Berger 2004; Qualité and Tillé 2008; Wood 2008; Muennich and Zins 2011).

Berger and Priam $(2010,2015)$ proposed a multivariate approach to estimate the correlation between functions of totals by incorporating the information related to the whole sample, $s=s_{1} \cup s_{2}$. This approach can be used to estimate the variance of change between poverty rates when we ignore the sampling variability due to the estimated poverty threshold $0.6 \hat{Y}_{t ; 0.5}$, that is, when we treat the poverty rates as simple ratios.

When we treat the threshold as fixed, the change becomes a smooth function of four totals, that is, $\hat{\Delta}=g(\hat{\tau})$, where $\hat{\tau}=\left(\hat{\tau}_{1}, \hat{\tau}_{2}, \hat{\tau}_{3}, \hat{\tau}_{4}\right)^{\top}$ is a vector of four estimated totals. Berger and Priam $(2010,2015)$ showed that using the first-order Taylor approximation, the design-based variance of $\hat{\Delta}$ can be estimated by

$$
\widehat{\operatorname{var}}(\hat{\Delta})=\widehat{\operatorname{grad}}(\hat{\tau})^{\top} \widehat{\operatorname{var}}(\hat{\tau}) \widehat{\operatorname{grad}}(\hat{\tau})
$$

where $\widehat{\operatorname{grad}}(\hat{\tau})$ is the gradient of $g(\hat{\tau})$ evaluated at $\hat{\tau}$, that is,

$$
\widehat{\operatorname{grad}}(\hat{\tau})=\frac{\partial g(\hat{\tau})}{\partial \hat{\tau}}=\left(-\frac{1}{\hat{\tau}_{2}},-\frac{\hat{\tau}_{1}}{\hat{\tau}_{2}^{2}}, \frac{1}{\hat{\tau}_{3}},-\frac{\hat{\tau}_{3}}{\hat{\tau}_{4}^{2}}\right)^{\top}
$$

and $\widehat{\operatorname{var}}(\hat{\tau})$ is given by

$$
\widehat{\operatorname{var}}(\hat{\tau})=\hat{\boldsymbol{D}}^{\top} \hat{\mathbf{\Sigma}} \hat{\boldsymbol{D}}
$$

with

$$
\hat{\boldsymbol{D}}=\operatorname{diag}\left\{\sqrt{\widehat{\operatorname{var}}\left(\hat{\tau}_{1}\right) \hat{\Sigma}_{11}^{-1}}, \sqrt{\widehat{\operatorname{var}}\left(\hat{\tau}_{2}\right) \hat{\Sigma}_{22}^{-1}}, \sqrt{\widehat{\operatorname{var}}\left(\hat{\tau}_{3}\right) \hat{\Sigma}_{33}^{-1}}, \sqrt{\widehat{\operatorname{var}}\left(\hat{\tau}_{4}\right) \hat{\Sigma}_{44}^{-1}}\right\},
$$

where $\hat{\boldsymbol{\Sigma}}$ is the Ordinary Least Square (OLS) estimator of the residual covariance matrix $\boldsymbol{\Sigma}$ 
of the multivariate linear regression Model in (3) proposed by Berger and Priam (2010, 2015); $\widehat{\operatorname{var}}\left(\hat{\tau}_{k}\right)$ is the design-based variance estimator of the Horvitz and Thompson (1952) estimator of total $\tau_{k}$, and $\hat{\Sigma}_{k k}^{-1}$ is the $k$-th diagonal element of $\hat{\boldsymbol{\Sigma}}(k=1,2,3,4)$. Berger and Priam $(2010,2015)$ showed that (2) gives an approximately unbiased estimator for the variance of change.

Let $\breve{p}_{t ; i}=\delta\left\{y_{t ; i} \leq 0.6 \hat{Y}_{t ; 0.5}\right\} \pi_{t ; i}^{-1}$ and $w_{t ; i}=\pi_{t ; i}^{-1}$. The multivariate model is given as follows,

$$
\left(\begin{array}{c}
\breve{p}_{1 ; i} \\
w_{1 ; i} \\
\breve{p}_{2 ; i} \\
w_{2 ; i}
\end{array}\right)=\left(\begin{array}{c}
\alpha_{1 ; 1} z_{1 ; i}+\alpha_{1 ; 2} z_{2 ; i}+\alpha_{1 ; 3} z_{1 ; i} \times z_{2 ; i} \\
\beta_{1 ; 1} z_{1 ; i}+\beta_{1 ; 2} z_{2 ; i}+\beta_{1 ; 3} z_{1 ; i} \times z_{2 ; i} \\
\alpha_{2 ; 1} z_{1 ; i}+\alpha_{2 ; 2} z_{2 ; i}+\alpha_{2 ; 3} z_{1 ; i} \times z_{2 ; i} \\
\beta_{2 ; 1} z_{1 ; i}+\beta_{2 ; 2} z_{2 ; i}+\beta_{2 ; 3} z_{1 ; i} \times z_{2 ; i}
\end{array}\right)+\boldsymbol{\epsilon}_{i}
$$

The vector of the residuals $\boldsymbol{\epsilon}_{\boldsymbol{i}}$ follow a multivariate distribution with mean $\mathbf{0}$ and covariance $\boldsymbol{\Sigma}$. Rotation of the sampling design is incorporated into the model through the model covariates: $z_{t ; i}=\delta\left\{i \in s_{t}\right\}$ and $z_{1 ; i} \times z_{2 ; i}=\delta\left\{i \in s_{1}, i \in s_{2}\right\}$. It should be noted that the correlations $\widehat{\operatorname{corr}}\left(\hat{\tau}_{k}, \hat{\tau}_{\ell}\right)$, with $(k, \ell=1,2,3,4)$, are obtained from the estimated residual covariance matrix $\hat{\mathbf{\Sigma}}$. The covariance terms on the nondiagonal part of the matrix $\widehat{\operatorname{var}}(\hat{\tau})$ are based on those estimated correlations $\widehat{\operatorname{corr}}\left(\hat{\tau}_{k}, \hat{\tau}_{\ell}\right)$ and the estimated cross-sectional variance terms $\widehat{\operatorname{var}}\left(\hat{\tau}_{k}\right)$. Note that this approach also accounts for a multistage sampling, using an "ultimate cluster approach" (e.g., Osier et al. 2013; Di Meglio et al. 2013).

Berger and Priam $(2010,2015)$ showed that the multivariate approach gives estimates which are approximately equal to the Hansen and Hurwitz (1943) variance estimator (e.g., Holmes and Skinner 2000).

The approach proposed can be easily extended to a stratified sampling. In this case, we assume that the sample sizes within each stratum are fixed (nonrandom) quantities. The model covariates $z_{t ; i}$ are replaced by the stratum wave indicators $z_{t h ; i}=\delta\left\{i \in s_{t h}\right\}$, where $s_{t h}$ is the sample for the stratum $h$ at wave $t$. As the rotation is done within each stratum, we consider the interactions $z_{t h ; i} \times z_{(t+1) h ; i}$.

\section{Allowing for the Variability of the Poverty Threshold}

Note that in (2), the variability of the poverty threshold is not taken into account because we treat $\hat{\theta}_{1}$ and $\hat{\theta}_{2}$ as ratios. Treating the poverty threshold as fixed might lead to an overestimation of the variances (e.g., Preston 1995; Berger and Skinner 2003; Verma and Betti 2011). Verma and Betti (2011) compared the ratio variance estimator (i.e., when the poverty threshold is treated as fixed) with linearisation and jackknife repeated replication. They found that the ratio variance estimator overestimated the standard errors for all the poverty measures and several complex statistics. However, these findings are related to the cross-sectional estimators and do not necessarily hold for the variance of change.

Taking into account the whole variability means that the sampling variation of the poverty threshold is also considered. However, the poverty rate is more complex than a ratio and cannot be expressed as a function of totals. We propose using the linearisation approach proposed by Deville (1999). The implementation of this approach for the poverty rate and 
the inequality measures can be found in the literature (e.g., Berger and Skinner 2003; Verma and Betti 2005; Osier 2009; Muennich and Zins 2011; Verma and Betti 2011).

The linearised variable $L_{t ; i}$ for individual $i$ at wave $t$ for the poverty rate is given by (see Osier 2009)

$$
L_{t ; i}=\frac{1}{\hat{N}_{t}}\left(\delta\left\{y_{t ; i} \leq 0.6 \hat{Y}_{t ; 0.5}\right\}-\hat{\theta}_{t}\right)-\frac{0.6}{\hat{N}_{t}} \frac{\hat{f}_{t}\left(0.6 \hat{Y}_{t ; 0.5}\right)}{\hat{f}_{t}\left(\hat{Y}_{t ; 0.5}\right)}\left(\delta\left\{y_{t ; i} \leq \hat{Y}_{t ; 0.5}\right\}-0.5\right),
$$

where $\hat{f}_{t}($.$) is an estimator of the density function, which is defined in (5). The second term$ in (4) is an additional term which reflects the sample variation originating from the randomness of the estimated median income.

The density functions can be estimated on the basis of the Gaussian kernel function as follows (e.g., Preston 1995):

$$
\hat{f}_{t}(x)=\frac{1}{\hat{N}_{t} \hat{h}_{t}} \sum_{i \in s_{t}} \frac{1}{\pi_{t ; i}} K\left(\frac{x-y_{t ; i}}{\hat{h}_{t}}\right),
$$

where $K(\eta)=(\sqrt{2 \pi})^{-1} \exp \left(-\eta^{2} / 2\right)$ is the Gaussian kernel, $\hat{N}_{t}=\sum_{i \in s_{t}} \pi_{t ; i}^{-1}$ is the Horvitz and Thompson (1952) estimator of the population size at wave $t(t=1,2)$, and $\hat{h}_{t}$ is the bandwidth parameter, which can be defined in several ways (Silverman 1986, 45-48). For a normally distributed population and smooth densities, the following bandwidth parameter was recommended by Silverman $(1986,46)$ :

$$
\hat{h}_{t}=1.06 \hat{\sigma}_{t ; \hat{Y}} \hat{N}_{t}^{-1 / 5}
$$

where

$$
\hat{\sigma}_{t ; \hat{Y}}=\sqrt{\frac{1}{\hat{N}_{t}}\left\{\sum_{i \in s_{t}} \frac{1}{\pi_{t ; i}} y_{t ; i}^{2}-\frac{1}{\hat{N}_{t}}\left(\sum_{j \in s_{t}} \frac{1}{\pi_{t ; j}} y_{t ; j}\right)^{2}\right\}}
$$

is the estimated standard deviation of the income distribution. However, for skewed and long-tailed distributions, Silverman $(1986,47)$ proposed using the interquartile range instead of the standard deviation of the distribution, that is,

$$
\hat{h}_{t}=0.79 \hat{Y}_{t ; i q r} \hat{N}_{t}^{-1 / 5},
$$

where $\hat{Y}_{t ; i q r}=\hat{Y}_{t ; 0.75}-\hat{Y}_{t ; 0.25}$ is the weighted interquartile range of the income distribution. Another bandwidth, which is very suitable for many densities, even for the modest bimodal ones, was suggested by Silverman $(1986,48)$ as follows:

$$
\hat{h}_{t}=0.9 \hat{A}_{t} \hat{N}_{t}^{-1 / 5}
$$

where $\hat{A}_{t}=\min \left(\hat{\sigma}_{t ; \hat{Y}}, \hat{Y}_{t ; i q r} / 1.34\right)$. It should be noted that the bandwidth in (8) is smaller than the other bandwidths in (6) and (7). Thus we are likely to obtain less smooth densities with the bandwidth (8).

It is worth mentioning that choosing a bandwidth parameter is a crucial step in applications (e.g., Verma and Betti 2005; Graf 2013; Graf and Tillé 2014). For example, Verma and Betti (2005) showed that probability density functions are sensitive to the 
chosen bandwidth parameter. A large value for the bandwidth parameter results in a smoother density. Graf (2013, 26-28) pointed out the potential danger of using standard deviation when estimating densities that might arise from extreme values in the data observed (for example, with income data). In such cases, Graf (2013) proposed using the logarithm to reduce the adverse impact of extreme values. He also remarked the fixedbandwidth parameter might be problematic when observations are heaped up around some values. To avoid this problem, a more robust technique to estimate density involving nearest neighbours with minimal bandwidth was suggested by Graf (2013).

\section{Estimation of Change Within Domains}

In practice, we are often interested in change within domains of interest. For example, we may be interested in change in poverty within different age groups. According to the definition given by Eurostat (2003), the poverty threshold is calculated based on the overall estimated median income rather than the estimated median income within the domains. Hence, when we are interested in a domain, the threshold will be the same for all domains.

Consider $d_{t ; i}$ to be a domain indicator for individual $i$ at wave $t$ defined by

$$
d_{t ; i}= \begin{cases}1 & \text { if } i \in D \text { at wave } t \\ 0 & \text { if } i \notin D \text { at wave } t\end{cases}
$$

where $D$ refers to the domain of interest. The poverty rate over a domain is defined by

$$
\hat{\theta}_{D t}=\frac{\sum_{i \in s_{t}} d_{t ; i} \delta\left\{y_{t ; i} \leq \hat{Y}_{t ; 0.5}\right\} \pi_{t ; i}^{-1}}{\sum_{i \in s_{t}} d_{t ; i} \pi_{t ; i}^{-1}}
$$

To estimate the variance of change within domains under the ratio approach (see (2)), we substitute $\breve{p}_{t ; i}$ by $\breve{p}_{D t ; i}=d_{t ; i} \breve{p}_{t ; i}$, and $\omega_{t ; i}$ by $\omega_{D t ; i}=d_{t ; i} \omega_{t ; i}$ in the model in (3). Note that the values of the response variables will be equal to zero for the units not included in the domain of interest.

For the linearisation approach, the linearised variables $L_{D t ; i}$ for individual $i$ in domain $D$ at wave $t$ derived in Appendix B (see B.5) are given by

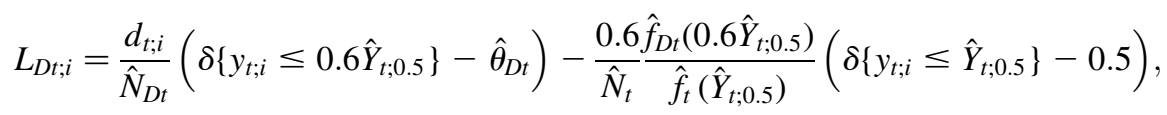

where

$$
\begin{gathered}
\hat{N}_{D t}=\sum_{i \in s_{t}} \frac{d_{t ; i}}{\pi_{t ; i}} \\
\hat{f}_{D t}(x)=\frac{1}{\hat{N}_{D t} \hat{h}_{D t}} \sum_{i \in s_{t}} \frac{d_{t ; i}}{\pi_{t ; i}} K_{D}\left(\frac{x-y_{t ; i}}{\hat{h}_{D t}}\right) .
\end{gathered}
$$

Here, $\hat{h}_{D t}$ can be (6), (7), or (8) with $\hat{N}_{D t}, \hat{Y}_{D t ; i q r}=\hat{Y}_{D t ; 0.75}-\hat{Y}_{D t ; 0.25 \text {, }}$ 


$$
\hat{\sigma}_{D t ; \hat{Y}}=\sqrt{\frac{1}{\hat{N}_{D t}}\left\{\sum_{i \in s_{t}} \frac{d_{t ; i}}{\pi_{t ; i}} y_{t ; i}^{2}-\frac{1}{\hat{N}_{D t}}\left(\sum_{j \in s_{t}} \frac{d_{t ; j}}{\pi_{t ; j}} y_{t ; j}\right)^{2}\right\}}
$$

and $\hat{A}_{D t}=\min \left(\hat{\sigma}_{D t ; \hat{Y}}, \hat{Y}_{D t ; i q r} / 1.34\right)$. Let $\hat{\Delta}_{D}=\hat{\theta}_{D 2}-\hat{\theta}_{D 1}$ be the change in poverty rate in domain $D$ between Wave 1 and Wave 2. Thus the variance of domain change is estimated by

$$
\widehat{\operatorname{var}}\left(\hat{\Delta}_{D}\right)=\widehat{\operatorname{var}}\left(\hat{\theta}_{D 1}^{L}\right)+\widehat{\operatorname{var}}\left(\hat{\theta}_{D 2}^{L}\right)-2 \widehat{\operatorname{corr}}\left(\hat{\theta}_{D 1}^{L}, \hat{\theta}_{D 2}^{L}\right) \sqrt{\widehat{\operatorname{var}}\left(\hat{\theta}_{D 1}^{L}\right) \widehat{\operatorname{var}}\left(\hat{\theta}_{D 2}^{L}\right)},
$$

with

$$
\hat{\theta}_{D t}^{L}=\sum_{i \in s_{t}} \frac{L_{D t ; i}}{\pi_{t ; i}}
$$

We use the approach proposed by Berger and Priam $(2010,2015)$ by treating $\hat{\theta}_{D 1}^{L}$ and $\hat{\theta}_{D 2}^{L}$ in (10) as the estimators of totals. The correlation term $\operatorname{corr}\left(\hat{\theta}_{D 1}^{L}, \hat{\theta}_{D 2}^{L}\right)$ in (9) is computed from the estimated residual covariance matrix $\hat{\mathbf{\Sigma}}$ of the following model,

$$
\left(\begin{array}{l}
\breve{L}_{D 1 ; i} \\
\breve{L}_{D 2 ; i}
\end{array}\right)=\left(\begin{array}{l}
\alpha_{1 ; 1} z_{1 ; i}+\alpha_{1 ; 2} z_{2 ; i}+\alpha_{1 ; 3} z_{1 ; i} \times z_{2 ; i} \\
\alpha_{2 ; 1} z_{1 ; i}+\alpha_{2 ; 2} z_{2 ; i}+\alpha_{2 ; 3} z_{1 ; i} \times z_{2 ; i}
\end{array}\right)+\boldsymbol{\epsilon}_{i},
$$

with $\breve{L}_{D t ; i}=L_{D t ; i} \pi_{t ; i}^{-1}$.

It should be noted that the domain information is incorporated into the model through the response variables, in contrast to the stratification (see Section 3). Note that the approach proposed can be used for strata domains and unplanned domains.

\section{Simulation Study}

In this section, the variance estimators from the ratio and the linearisation approaches are compared in terms of the relative bias (RB) and the root mean square error (RRMSE), respectively defined by (11) and (12). Additionally, we investigate whether the ratio approach gives more conservative estimates.

The income variables at Wave 1 and Wave 2 were generated according to different probability distributions (see Appendix A). For each wave, a gamma distribution ( shape $=2.5$, rate $=1$ ), a lognormal distribution (mean $=1.119$, standard deviation $=0.602$ ) and a Weibull distribution ( shape $=0.8$, scale $=1$ ) were used to generate populations with a size of $N=20,940$. As stated by Salem and Mount (1974) and McDonald (1984), these distributions are good approximations of income distributions. The correlation coefficient between the variables of the first and the second wave is given by $\rho=0.94$, which is the correlation observed from the common sample of the Turkish EU-SILC survey data. Note that this correlation and the correlation in (1) are different; in other words, the correlation $\rho=0.94$ is the correlation between the variables of interest, whereas the correlation in (1) is the correlation between the point estimators. 
The population is assumed fixed and the same sample size was used for both waves. We have 1,047 primary sampling units in the Turkish EU-SILC survey data. For this reason, we used $n_{1}=n_{2}=1,047$ units for each wave. The fraction of the common sample is 75 percent. Hence, the number of units in the common sample is $n_{c}=785$. Unequal and equal probabilities were used to select the samples. The Chao (1982) sampling design was used as unequal probability sampling $(\pi \mathrm{ps})$ design. The first-wave samples were selected without replacement with the inclusion probabilities proportional to a size variable $x_{i}$, which was generated by the model $x_{i}=\alpha+\rho y_{1 ; i}+e_{i}$, with $e_{i} \sim$ $N\left(0,\left(1-\rho^{2}\right) \sigma_{y 1}^{2}\right), \alpha=5$ and $\rho=0.7$. For the second wave, a simple random sample of $n_{c}$ units were selected from the sample $s_{1}$; and $n_{2}-n_{c}$ units were selected with the probabilities proportional to size $q_{i}=\pi_{1 ; i} /\left(1-\pi_{1 ; i}\right)$ from the population $U \backslash s_{1}$. It can be shown that $\pi_{2 ; i} \approx \pi_{1 ; i}$ (Christine and Rocher 2012). For equal probability sampling designs, $\pi_{2 ; i}=\pi_{1 ; i}=n_{1} / N$.

We did six simulation studies for three populations and two sampling designs. For each simulation, 10,000 samples were selected. For each sample, the RB and the RRMSE were computed for the cross-sectional variance estimators, the variance estimator of change and the estimator of the correlation. The RB and the RRMSE are defined by

$$
\begin{gathered}
R B(\hat{\sigma})=\frac{E(\hat{\sigma})-\sigma}{\sigma} 100 \%, \\
\operatorname{RRMSE}(\hat{\sigma})=\frac{\sqrt{(B-1)^{-1} \sum_{b=1}^{\mathrm{B}}\left(\hat{\sigma}_{b}-\sigma\right)^{2}}}{\sigma} 100 \%,
\end{gathered}
$$

where $E(\hat{\sigma})=B^{-1} \sum_{b=1}^{B} \hat{\sigma}_{b}$, with $B=10,000$, is the empirical expectation; $\sigma$ is either the empirical variance or the empirical correlation in (1); $\hat{\sigma}$ is the estimator of the quantity $\sigma$; $\hat{\sigma}_{b}$ is the estimate of the quantity $\sigma$ for the $b$-th sample. For the linearisation, we considered three bandwidth parameters (see (6), (7) and (8)). The linearisations based on (6), (7) and (8) are respectively labelled Lin_Sd, Lin_Iqr, and Lin_A in Table 1 and Table 2.

For a gamma distribution, the poverty rates are 24.2 percent and 23.6 percent for the first and the second wave respectively. Hence, we have -0.59 percentage point change between two waves. For a lognormal distribution, the poverty rates are 19.4 percent and 19.9 percent. Thus there is a 0.54 percentage point change in this case. For a Weibull distribution, we have the highest poverty rates, which are 36.6 percent and 37.3 percent respectively. Hence, the change is 0.66 percentage points.

Table 1 shows the RB (\%) of the variance and the correlation estimators for several distributions and sampling designs. Overall, the linearisation approach has lower RB compared to the ratio approach. Thus we achieve more accurate estimates with linearisation. Differences between the two approaches in terms of the RB are much more pronounced for the Weibull distribution, which is the most skewed distribution. For all situations except with the lognormal distribution, the ratio approach overestimates all the variances and the correlations. Therefore, the ratio approach may not always provide more conservative estimates. However, note that whenever we have a positive bias, we obtain relatively larger variance estimates with the ratio approach. When we compare the three linearisation methods based on different bandwidth, we obtained the largest RB with the smallest bandwidth (see (8)). 


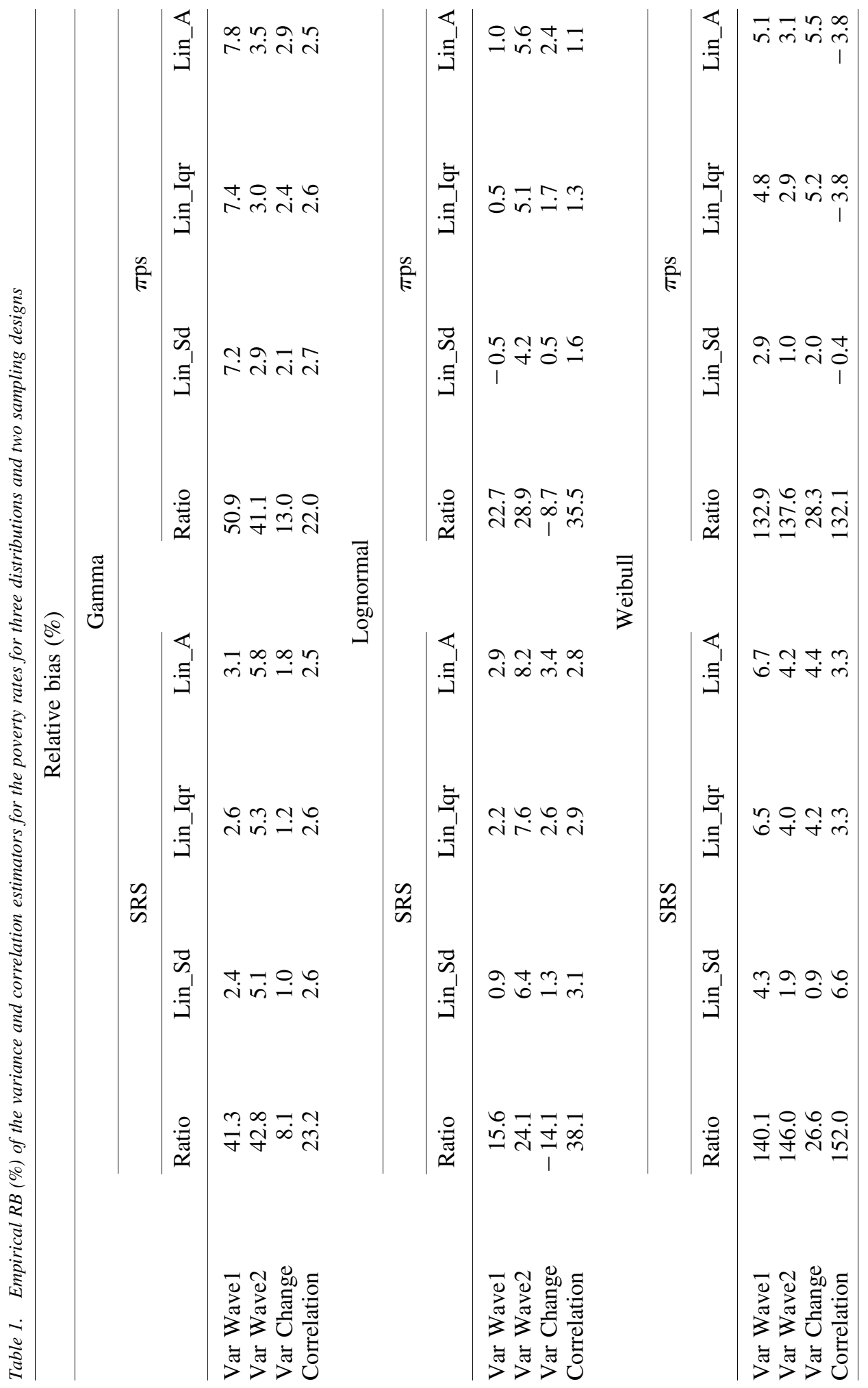


As far as the RRMSE is concerned (see Table 2), we achieve more precise estimates with the linearisation approach. We observe the smallest RRMSE with bandwidth (6) and the largest RRMSE with bandwidth (8). The ratio approach provides less accurate point estimates. However, the differences between the two approaches can be negligible for the variance of change, except with the Weibull distribution.

\section{An Application to the Turkish EU-SILC Survey}

The 2007 and 2008 cross-sectional Turkish EU-SILC survey data was used. The Turkish EU-SILC survey has a stratified two-stage cluster probability sampling design. For the first stage, address blocks are selected within each stratum with a probability proportional to size $(\pi \mathrm{ps})$ without replacement sampling design. Each block is composed of approximately 100 addresses. Households within the selected address blocks are selected using a systematic sampling design. All individuals within the selected households participate in the survey. The cross-sectional survey weights in the "personal register" file (RB050) were used as inverses of the inclusion probabilities. The effect of calibration was not taken into account because we did not have any information about the auxiliary variables. The effect of imputation was ignored for the same reason.

Table 2. Empirical RRMSE (\%) of the variance and correlation estimators for the poverty rates for three distributions and two sampling designs

\begin{tabular}{|c|c|c|c|c|c|c|c|c|}
\hline \multicolumn{9}{|c|}{ Relative root mean square error $(\%)$} \\
\hline & \multicolumn{8}{|c|}{ Gamma } \\
\hline & \multicolumn{4}{|c|}{ SRS } & \multicolumn{4}{|c|}{$\pi \mathrm{ps}$} \\
\hline & Ratio & Lin_Sd & Lin_Iqr & Lin_A & Ratio & Lin_Sd & Lin_Iqr & Lin_A \\
\hline Var Wave1 & 41.5 & 4.8 & 5.1 & 5.9 & 51.2 & 8.2 & 8.4 & 9.0 \\
\hline Var Wave2 & 36.8 & 6.8 & 7.1 & 7.9 & 41.4 & 4.9 & 5.1 & 5.9 \\
\hline Var Change & 10.9 & 7.9 & 8.1 & 8.6 & 15.4 & 8.7 & 8.8 & 9.4 \\
\hline \multirow[t]{4}{*}{ Correlation } & 20.0 & 6.0 & 6.5 & 6.5 & 22.7 & 7.3 & 7.3 & 7.3 \\
\hline & \multicolumn{8}{|c|}{ Lognormal } \\
\hline & \multicolumn{4}{|c|}{ SRS } & \multicolumn{4}{|c|}{$\pi \mathrm{ps}$} \\
\hline & Ratio & Lin_Sd & Lin_Iqr & Lin_A & Ratio & Lin_Sd & Lin_Iqr & Lin_A \\
\hline Var Wave1 & 16.4 & 4.9 & 6.2 & 7.2 & 30.6 & 7.8 & 8.2 & 8.7 \\
\hline Var Wave2 & 24.6 & 8.1 & 9.8 & 10.8 & 35.2 & 8.9 & 9.8 & 10.5 \\
\hline Var Change & 15.1 & 7.0 & 8.0 & 8.8 & 18.5 & 10.6 & 11.2 & 11.7 \\
\hline \multirow[t]{4}{*}{ Correlation } & 38.5 & 7.1 & 7.0 & 7.0 & 37.4 & 11.1 & 11.0 & 11.0 \\
\hline & \multicolumn{8}{|c|}{ Weibull } \\
\hline & \multicolumn{4}{|c|}{ SRS } & \multicolumn{4}{|c|}{$\pi \mathrm{ps}$} \\
\hline & Ratio & Lin_Sd & Lin_Iqr & Lin_A & Ratio & Lin_Sd & Lin_Iqr & Lin_A \\
\hline Var Wave1 & 140.1 & 6.5 & 8.5 & 9.0 & 133.0 & 6.5 & 8.0 & 8.5 \\
\hline Var Wave2 & 146.0 & 5.5 & 7.1 & 7.7 & 137.7 & 6.2 & 7.4 & 7.9 \\
\hline Var Change & 27.1 & 5.7 & 7.8 & 8.4 & 29.1 & 7.0 & 9.3 & 9.9 \\
\hline Correlation & 152.2 & 16.7 & 16.3 & 16.6 & 132.4 & 16.2 & 17.2 & 17.5 \\
\hline
\end{tabular}




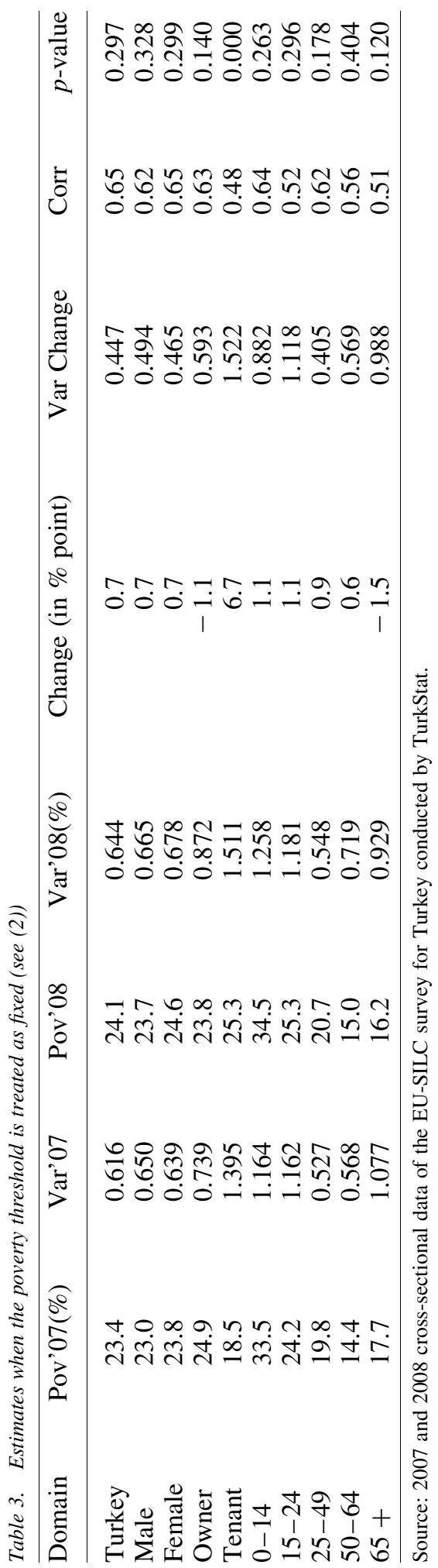




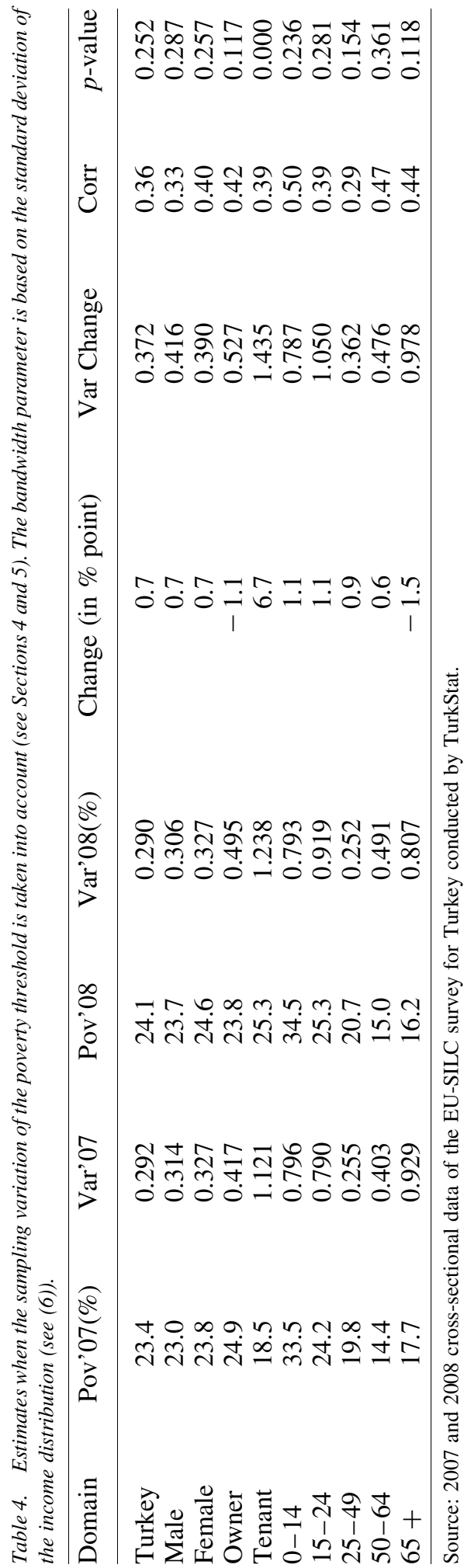




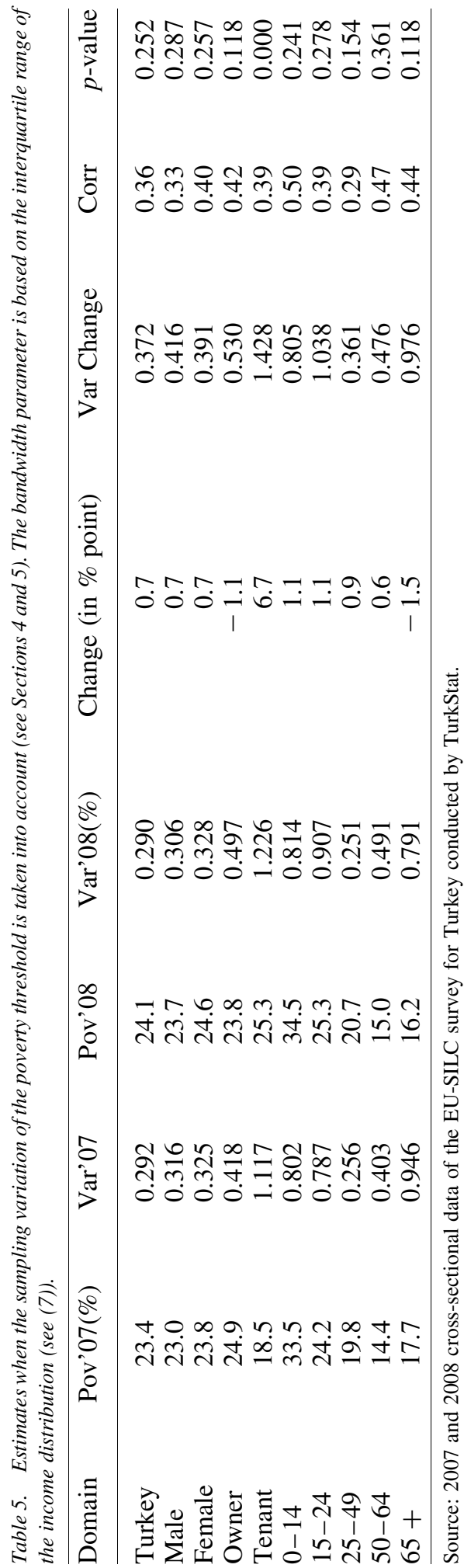




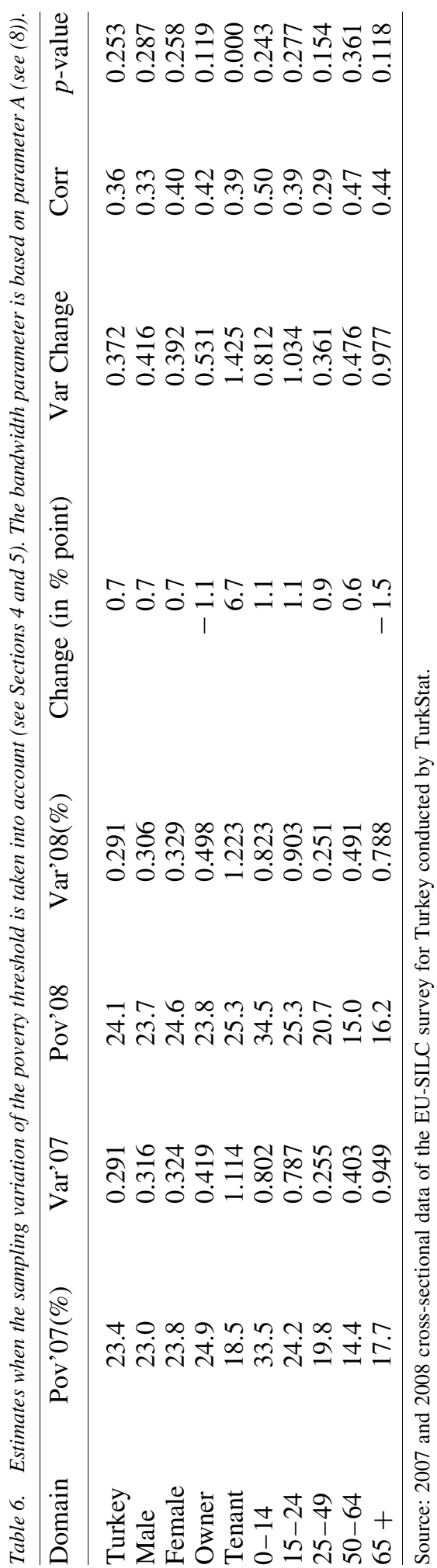


In Table 3, we give the estimates for several domains when the poverty threshold is treated as fixed (see (2)). We observe a significant change for the domain "tenant" at the 5 percent level.

In Table 4, we give the estimates obtained with the linearisation approach based on the bandwidth in (6) described in Section 4. Here, we again observe a highly significant change for the domain "tenant". We do not observe major differences in the $p$-values between Table 3 and Table 4 . We observe a slight decrease in the $p$-values when the sampling variation of the poverty threshold is taken into account. This is due to the fact that the variances of changes are larger in Table 3.

The correlations in Table 4 are smaller than in Table 3 overall. Hence, the estimated correlations are smaller when the variability of the poverty threshold is taken into account.

The comparison of Table 3 and Table 4 also revealed that all variances were estimated more conservatively when the threshold is treated as fixed. Preston (1995), Berger and Skinner (2003), and Verma and Betti (2011) demonstrated that the cross-sectional variances are more conservative when the poverty threshold is treated as fixed. This finding was explained by Preston (1995) by the fact that the two sources of variability offset each other. This is more pronounced when the high fractions of the median are used.

For the variance of change, we cannot anticipate an increase in the variance when the poverty threshold is treated as fixed for the following reason. Let us assume that the crosssectional variances are equal: $\widehat{\operatorname{var}}\left(\hat{\theta}_{1}\right)=\widehat{\operatorname{var}}\left(\hat{\theta}_{2}\right)$. Thus the variance estimator of change is given by $\widehat{\operatorname{var}}\left(\hat{\Delta}_{1}\right)=2 \widehat{\operatorname{var}}\left(\hat{\theta}_{1}\right)\left(1-\widehat{\operatorname{corr}}\left(\hat{\theta}_{1}, \hat{\theta}_{2}\right)\right)$. Hence, the variance of change is affected in the same direction by the variance term, and in the opposite direction by the correlation term. Accordingly, when both the variance and the correlation terms increase or decrease concurrently, the direction of the effect on the variance of change cannot be predicted. Therefore, we may not necessarily have more conservative estimates of the variance of change when the poverty threshold is treated as fixed. With the Turkish EU-SILC survey data, we found that the variances of changes were more conservative, although the differences between the two approaches were not as pronounced as the differences between the cross-sectional variances (see Table 3 and Table 4).

In Table 4, the bandwidth parameter is given by (6). We also investigate the situations when the bandwidth parameter is given by (7) and (8). The results are given in Table 5 and Table 6. By comparing Table 5 and Table 6 with Table 3, we also observed smaller cross-sectional variances, variance of change and correlation when the bandwidth parameter is (7) and (8). When we compare Table 4, Table 5, and Table 6, the estimates do not differ significantly between the three linearisation approaches based on different bandwidth parameters, although we observe slight differences between them in terms of the RB and the RRMSE in the simulation study (see Section 6).

\section{Conclusion}

We applied a simple approach to estimate the variances of changes for the poverty rates over several domains by using the 2007-2008 Turkish EU-SILC survey data. Our approach involves a multivariate linear regression model proposed by Berger and Priam (2010, 2015), which can be easily applied. Survey characteristics such as rotation, stratification, and cluster sampling are all taken into account. The approach proposed is flexible and can be implemented for most of the EU-SILC surveys as long as sampling 
fractions are negligible. This assumption implies that the second-order inclusion probabilities are not needed.

We have two ways of estimating the variances, depending on whether we treat the poverty threshold as fixed or not. When treated as fixed, we obtained more conservative variance estimates of change with the Turkish EU-SILC survey data. However, our simulation study shows that treating the threshold as fixed does not necessarily provide more conservative variance estimates of change. For the lognormal distribution, for example, variances of changes were underestimated with the ratio method. On the other hand, differences between the variance estimators of changes can be negligible in terms of the RB and the RRMSE, even though we observed significant differences between the cross-sectional variances and the correlations. For the latter, the linearisation approach gave more unbiased and more precise variance estimates. Thus based upon our results and due to the fact that linearisation involves complex numerical computations, the simple ratio approach may appear preferable to estimate the variance of change for the poverty rates. However, we should be careful with highly skewed distributions similar to a Weibull one. In this case, the linearisation approach is significantly better.

The approach proposed can also be used to estimate the variances of the other poverty and income inequality measures such as the relative median at-risk-of-poverty gap (RMPG), the quantile share ratio (QSR) and the Gini coefficient, which are included in the "Laeken" indicators (Eurostat 2003), by using linearisation (e.g., Berger 2008). The RMPG and the Gini coefficient can not be treated as a simple ratio, whereas the QSR can be. The linearised variables of many complex parameters are given by Verma and Betti $(2005,2011)$.

In this article, we implemented the fixed-bandwidth kernel method for its simplicity (Silverman 1986, 95). Note that the bandwidth in (8) is a suitable choice for a wide range of densities, as pointed out by Silverman (1986). If the distribution is heavily skewed, then an adaptive kernel method can be applied (Silverman 1986, chap. 5). This method uses a variable bandwidth, that is, for each observed data point, a different bandwidth is computed. It would be interesting to check whether an adaptive bandwidth improved the variance estimation in the presence of outliers.

\section{Appendix A. Generation of the Income Variables for the Simulation Study}

For the gamma random variables, we used the algorithm proposed by Schmeiser and Lal (1982, 358). First, three independent random variables were generated by a gamma distribution as follows:

$$
\begin{aligned}
& Y_{1} \sim \operatorname{Gamma}\left(\alpha_{1}-\rho \sqrt{\alpha_{1}} \sqrt{\alpha_{2}}, 1\right), \\
& Y_{2} \sim \operatorname{Gamma}\left(\alpha_{2}-\rho \sqrt{\alpha_{1}} \sqrt{\alpha_{2}}, 1\right), \\
& Y_{3} \sim \operatorname{Gamma}\left(\rho \sqrt{\alpha_{1}} \sqrt{\alpha_{2}}, 1\right),
\end{aligned}
$$

with $\alpha_{1}=2.5, \alpha_{2}=2.6$, and $\rho=0.94$. Then, the income variables were obtained by the following expressions: $y_{1 ; i}=Y_{1}+Y_{3}$ and $y_{2 ; i}=Y_{2}+Y_{3}$, so that $y_{1 ; i} \sim \operatorname{Gamma}(2.5,1)$, $y_{2 ; i} \sim \operatorname{Gamma}(2.6,1)$, and $\rho\left(y_{1 ; i}, y_{2 ; i}\right) \approx 0.94$.

The Cholesky decomposition was used to generate the correlated lognormal variables. Hence, the $\log$ income variables with the correlation of $\rho=0.95$, a mean of $\mu=1.119$ 
and a standard deviation of $\sigma=0.602$ were generated by

$$
\begin{gathered}
\log \left(y_{1 ; i}\right)=\mu+\sigma X_{1} \\
\log \left(y_{2 ; i}\right)=\mu+\rho \sigma X_{1}+\sqrt{1-\rho^{2}} \sigma X_{2},
\end{gathered}
$$

where $X_{1}$ and $X_{2}$ are independent standard normal variables. The correlation coefficient between the income variables was approximately 0.94 .

For correlated Weibull variables, we followed the algorithm proposed by Feiveson (2002, 117). Firstly, two correlated standard normal variables $Y_{1}$ and $Y_{2}$ with a correlation of $\rho=0.95$ were generated by using the Cholesky decomposition: $Y_{1}=X_{1}$ and $Y_{2}=\rho X_{1}+\sqrt{1-\rho^{2}} X_{2}$, where $X_{1}$ and $X_{2}$ are independent standard normal variables. Secondly, correlated uniform variables were obtained by the standard normal cumulative distribution function transformation; such that $U_{1}=\Phi\left(Y_{1}\right)$ and $U_{2}=\Phi\left(Y_{2}\right)$, where $\Phi(\cdot)$ is the cumulative distribution function of a standard normal distribution. Finally, uniform random variables were transformed by the inverse of the Weibull cumulative distribution function to achieve the correlated income variables as follows: $y_{1 ; i}=\mathrm{F}_{U}^{-1}\left(U_{1}\right)=\left(-\ln \left(1-U_{1}\right)\right)^{5 / 4}$ and $y_{2 ; i}=\mathrm{F}_{U}^{-1}\left(U_{2}\right)=\left(-\ln \left(1-U_{2}\right)\right)^{5 / 4}$, so that $y_{1 ; i}, y_{2 ; i} \sim \operatorname{Weibull}(0.8,1)$ and $\rho\left(y_{1 ; i}, y_{2 ; i}\right) \approx 0.94$.

\section{Appendix B. Derivation of the Influence Function of the Poverty Rate Over a Domain}

Let $\mathrm{M}$ be a measure that assigns a unit mass to each unit $i$ in the population $U$. For example, the population size $N$ can be written as $N=\int d \mathrm{M}=\sum_{i \in U} 1$ and the total of a variable $y$ can be expressed as $N=\int y d \mathrm{M}=\sum_{i \in U} y_{i}$ (Deville 1999). Let $\mathrm{F}(\mathrm{M}, x)$ be the income distribution function at $x$ over the population $U$, that is,

$$
\mathrm{F}(\mathrm{M}, x)=\frac{1}{N} \sum_{i \in U} \delta\left\{y_{i} \leq x\right\} .
$$

Then, the income distribution function at the median of the income distribution is given by $\mathrm{F}(\mathrm{M}, \operatorname{Med}(\mathrm{M}))=0.5$. Thus the influence function of the functional $\mathrm{F}(\mathrm{M}, \operatorname{Med}(\mathrm{M}))$ at $i$ is equal to 0 , that is, $\mathrm{IF}_{i}(\mathrm{M}, \operatorname{Med}(\mathrm{M}))=0$. By using "Rule 7" in Deville (1999, 198), the influence function of $\mathrm{F}$ at $i$ (see also Osier 2009, 181-183) can be derived as follows:

$$
\mathrm{IF}_{i}(\mathrm{M}, \operatorname{Med}(\mathrm{M}))=\mathrm{IF}_{i}\left(\mathrm{M},\left.\operatorname{Med}(\mathrm{M})\right|_{\operatorname{Med}(\mathrm{M}) \mathrm{fixed}}\right)+\left.\frac{\partial \mathrm{F}(\mathrm{M}, x)}{\partial x}\right|_{x=\operatorname{Med}(\mathrm{M})} \operatorname{IMed}_{i}(\mathrm{M})=0 .
$$

The influence function of $\mathrm{F}$, when the median is fixed, is given by

$$
\mathrm{IF}_{i}\left(\mathrm{M},\left.\operatorname{Med}(\mathrm{M})\right|_{\operatorname{Med}(\mathrm{M}) \text { fixed }}\right)=\frac{1}{N}\left[\delta\left\{y_{i} \leq \operatorname{Med}\right\}-0.5\right] .
$$

Thus the influence function of the functional $\operatorname{Med}(\mathrm{M})$ is obtained as

$$
\operatorname{IMed}_{i}(\mathrm{M})=-\frac{1}{N} \frac{1}{f(M e d)}\left[\delta\left\{y_{i} \leq \operatorname{Med}\right\}-0.5\right],
$$


where

$$
f(M e d)=\left.\frac{\partial \mathrm{F}(\mathrm{M}, x)}{\partial x}\right|_{x=\operatorname{Med}(\mathrm{M})}
$$

is the probability density function at the median of the income distribution.

Now define the income distribution function at $x$ over a domain $D$ as follows:

$$
\mathrm{F}_{D}(\mathrm{M}, x)=\frac{1}{N_{D}} \sum_{i \in U} d_{i} \delta\left\{y_{i} \leq x\right\} .
$$

Hence, the income distribution function over a domain $D$ at the poverty threshold $T$ is defined by

$$
\mathrm{F}_{D}(\mathrm{M}, T(\mathrm{M}))=\frac{1}{N_{D}} \sum_{i \in U} d_{i} \delta\left\{y_{i} \leq T(\mathrm{M})\right\}
$$

where $T(\mathrm{M})=0.6 \operatorname{Med}(\mathrm{M})$ and $d_{i}$ is the domain indicator, that is, 1 when $i \in D$, and 0 otherwise. $\mathrm{F}_{\mathrm{D}}(\mathrm{M}, T(\mathrm{M}))$ is equivalent to the poverty rate over a domain $D$ (i.e., $R_{D}$ ). Thus we can obtain the influence function of the poverty rate analogously to (B.1), that is,

$$
\left.\operatorname{IF}_{D ; i}(\mathrm{M}, T(\mathrm{M}))=\left.\operatorname{IF}_{D ; i}(\mathrm{M}, T(\mathrm{M}))\right|_{T(\mathrm{M}) \text { fixed }}\right)+\left.\frac{\partial \mathrm{F}_{D}(\mathrm{M}, x)}{\partial x}\right|_{x=T(\mathrm{M})} \operatorname{IT}_{i}(\mathrm{M})=\operatorname{IR}_{D ; i} .
$$

The influence function of $\mathrm{F}_{D}$, when the threshold is fixed, is given by

$$
\left.\mathrm{IF}_{D ; i}(\mathrm{M}, T(\mathrm{M}))\right|_{T(\mathrm{M}) \text { fixed }}=\frac{d_{i}}{N_{D}}\left[\delta\left\{y_{i} \leq T\right\}-R_{D}\right] .
$$

Hence, the influence function of the poverty rate is obtained as follows:

$$
\mathrm{IR}_{D ; i}=\frac{d_{i}}{N_{D}}\left[\delta\left\{y_{i} \leq T\right\}-R_{D}\right]+f_{D}(T) \operatorname{IT}_{i}(\mathrm{M}),
$$

where

$$
f_{D}(T)=\left.\frac{\partial \mathrm{F}_{D}(\mathrm{M}, x)}{\partial x}\right|_{x=T(\mathrm{M})}
$$

is the probability density function at the poverty threshold. The influence function of the functional $\mathrm{T}(\mathrm{M})$ at $i$ is given by

$$
\operatorname{IT}_{i}(\mathrm{M})=0.6 \operatorname{IMed}_{i}(\mathrm{M}) .
$$

If we substitute $\operatorname{IMed}_{i}(\mathrm{M})$ in (B.2) into (B.4), we obtain the following:

$$
\mathrm{IT}_{i}(\mathrm{M})=-\frac{0.6}{N} \frac{1}{f(\text { Med })}\left[\delta\left\{y_{i} \leq \text { Med }\right\}-0.5\right] .
$$

Therefore, the influence function of the poverty rate at $i$ over a domain $D$ given in (B.3) can be rewritten as follows:

$$
\mathrm{IR}_{D ; i}=\frac{d_{i}}{N_{D}}\left[\delta\left\{y_{i} \leq T\right\}-R_{D}\right]-\frac{0.6}{N} \frac{f_{D}(T)}{f(M e d)}\left[\delta\left\{y_{i} \leq M e d\right\}-0.5\right] .
$$

Note that we assume the derivatives of $\mathrm{F}$ and $\mathrm{F}_{D}$ exist and are strictly non-negative for all $x$. 


\section{References}

Atkinson, A.B. and E. Marlier. 2010. "Income and Living Conditions in Europe." Publications Office of the European Union, Luxembourg. Available at: http://ec.europa. eu/eurostat/documents/3217494/5722557/KS-31-10-555-EN.PDF/e8c0a679-be01-461ca08b-7eb08a272767 (accessed April 30, 2015).

Berger, Y.G. 2004. "Variance Estimation for Measures of Change in Probability Sampling." Canadian Journal of Statistics 32: 451-467. DOI: http://dx.doi.org/ $10.2307 / 3316027$.

Berger, Y.G. 2008. "A Note on the Asymptotic Equivalence of Jackknife and Linearization Variance Estimation for the Gini Coefficient." Journal of Official Statistics 24: 541-555.

Berger, Y.G., T. Goedemé, and G. Osier. 2013. Handbook on Standard Error Estimation and Other Related Sampling Issues in EU-SILC Second Network for the Analysis of EU-SILC, EuroStat. Available at: http://www.cros-portal.eu/content/handbook-standard-error-estimation-and-other-related-sampling-issues-ver-29072013 (accessed February 6, 2013).

Berger, Y.G. and R. Priam. 2010. "Estimation of Correlations between Cross-Sectional Estimates from Repeated Surveys - an Application to the Variance of Change." In Proceedings of the 2010 Symposium of Statistics Canada, [26-29 October, 2010]. [10 pp.].

Berger, Y.G. and R. Priam. 2015. A Simple Variance Estimator of Change for Rotating Repeated Surveys: an Application to the EU-SILC Household Surveys. Journal of the Royal Statistical Society, Series A (Statistics in Society). Available at: DOI: http:// dx.doi.org/10.1111/rssa.12116. 22 pp. (accessed June 9, 2015).

Berger, Y.G. and C.J. Skinner. 2003. "Variance Estimation of a Low-Income Proportion." Journal of the Royal Statistical Society, Series C (Applied Statistics) 52: 457-468. DOI: http://dx.doi.org/10.1111/1467-9876.00417.

Betti, G. and F. Gagliardi. 2007. "Jackknife Variance Estimation of Differences and Averages of Poverty Measures.” Working Paper 68, Siena: Dipartimento di Metodi Quantitativi, Università degli Studi.

Chao, M.T. 1982. "A General Purpose Unequal Probability Sampling Plan.” Biometrika 69: 653-656. DOI: http://dx.doi.org/10.1093/biomet/69.3.653.

Christine, M. and T. Rocher. 2012. "Construction d'échantillons astreints á des conditions de recouvrement par rapport un échantillon antérieur et á des conditions d'équilibrage par rapport á des variables courantes." Proceedings of the 10th Journée de Méthodologie Statistique de l'INSEE, January 24-26, 2012. [41 pp.]. Paris.

Demnati, A. and J.N.K. Rao. 2004. "Linearization Variance Estimators for Survey Data." Survey Methodology 30: 17-26.

Deville, J.C. 1999. "Variance Estimation for Complex Statistics and Estimators: Linearization and Residual Techniques.” Survey Methodology 25: 193-203.

Di Meglio, E., G. Osier, T. Goedemé, Y. G. Berger, and E. Di Falco. 2013. "Standard Error Estimation in EU-SILC - First Results of the Net-SILC2 Project.” In Proceedings of the Conference on New Techniques and Technologies for Statistics, [March 5-7, 
2013]. [10 pp.]. Brussels. Available at: http://www.crosportal.eu/sites/default/files// NTTS2013\%20Proceedings_0.pdf (last accessed April 30, 2015).

Eurostat. 2003. “'Laeken' Indicators-Detailed Calculation Methodology.” Available at: http://www.cso.ie/en/media/csoie/eusilc/documents/Laeken\%20Indicators\%20-\% 20calculation\%20algorithm.pdf (accessed February 4, 2014).

Eurostat. 2012. "European Union Statistics on Income and Living Conditions (EU-SILC).” Available at: http://epp.eurostat.ec.europa.eu/portal/page/portal/ microdata/eusilc (accessed January 7, 2013).

Feiveson, A.H. 2002. "Power by simulation." The STATA Journal 2: 107-124.

Gambino, J.G. and P.L.N. Silva. 2009. "Sampling and Estimation in Household Surveys." In Handbook of Statistics, 29A: Design, Method and Applications, edited by D. Pfeffermann and C.R. Rao, 407-439. Amsterdam: Elsevier.

Goedemé, T. 2010. "The Standard Error of Estimates Based on EU-SILC. An Exploration through the Europe 2020 Poverty Indicators." Working paper 10/09, [Herman Deleeck Centre for Social Policy, University of Antwerp, Belgium]. Available at: http://www. centrumvoorsociaalbeleid.be/index.php?q=node/2204/en (accessed April 30, 2015).

Graf, E. 2013. "Variance Estimation by Linearization for Indicators of Poverty and Social Exclusion in a Person and Household Survey Context." Paper presented at New Techniques and Technologies for Statistics, Brussels. Available at: http://www. cros-portal.eu/content/14a01 ericgraf (last accessed February 5, 2014).

Graf, E. and Y. Tillé. 2014. "Variance Estimation Using Linearization for Poverty and Social Exclusion Indicators.” Survey Methodology 40: 61-79.

Hansen, M.H. and W.N. Hurwitz. 1943. "On the Theory of Sampling from Finite Populations." The Annals of Mathematical Statistics 14: 333-362.

Holmes, D.J. and C.J. Skinner. 2000. "Variance Estimation for Labour Force Survey Estimates of Level and Change.” The Office for National Statistics, London, United Kingdom. Government Statistical Service Methodology Series 21, 40 pp.

Horvitz, D.G. and D.J. Thompson. 1952. "A Generalization of Sampling Without Replacement From a Finite Universe.” Journal of the American Statistical Association 47: 663-685. DOI: http://dx.doi.org/10.1080/01621459.1952.10483446.

Kalton, G. 2009. “Design for Surveys over Time.” In Handbook of Statistics, 29A: Design, Method and Applications, edited by D. Pfeffermann and C.R Rao, 89-108. Amsterdam: Elsevier.

Kish, L. 1965. Survey Sampling. New York: John Wiley and Sons.

Laniel, N. 1987. "Variances for a Rotating Sample from a Changing Population." In Proceedings of the Survey Research Methods Section, American Statistical Association, [August 17-20, 1987]. 496-500. Alexandria, VA: American Statistical Association.

McDonald, J.B. 1984. "Some Generalized Functions for the Size Distribution of Income." Econometrica 52: 647-664.

Muennich, R. and S. Zins. 2011. "Variance Estimation for Indicators of Poverty and Social Exclusion." Work package of the European project on Advanced Methodology for European Laeken Indicators (AMELI). Available at: http://www.uni-trier.de/index. php?id=24676 (accessed January 4, 2013). 
Nordberg, L. 2000. "On Variance Estimation for Measures of Change when Samples Are Coordinated by the Use of Permanent Random Numbers." Journal of Official Statistics 16: 363-378.

Osier, G. 2009. "Variance Estimation for Complex Indicators of Poverty and Inequality Using Linearization Techniques.” Survey Research Methods 3: 167-195.

Osier, G., Y.G. Berger, and T. Goedemé. 2013. "Standard Error Estimation for the EU-SILC Indicators of Poverty and Social Exclusion.” Eurostat Methodologies and Working Papers series. Publications Office of the European Union, Luxembourg. Available at: http://ec.europa.eu/eurostat/documents/3888793/5855973/KS-RA-13024-EN.PDF (accessed April 30, 2015).

Preston, I. 1995. "Sampling Distributions of Relative Poverty Statistics." Applied Statistics 44: 91-99.

Qualité, L. and Y. Tillé. 2008. "Variance Estimation of Changes in Repeated Surveys and its Application to the Swiss Survey of Value Added." Survey Methodology 34: 173-181.

Salem, A.B.Z. and T.D. Mount. 1974. "A Convenient Descriptive Model of Income Distribution: The Gamma Density." Econometrica 42: 1115-1127.

Schmeiser, B.W. and R. Lal. 1982. "Bivariate Gamma Random Vectors." Operations Research 30: 355-374. DOI: http://dx.doi.org/10.1287/opre.30.2.355.

Silverman, B.W. 1986. Density Estimation for Statistics and Data Analysis. London: Chapman and Hall.

Tam, S.M. 1984. "On Covariances from Overlapping Samples.” American Statistician 38: 288-289. DOI: http://dx.doi.org/10.1080/00031305.1984.10483227.

Verma, V. and G. Betti. 2005. "Sampling Errors and Design Effects for Poverty Measures and Other Complex Statistics.” Working Paper 53, Siena: Dipartimento di Metodi Quantitativi, Università degli Studi.

Verma, V. and G. Betti. 2011. "Taylor Linearisation Sampling Errors and Design Effects for Poverty Measures and Other Complex Statistics.” Journal of Applied Statistics 38: 1549-1576. DOI: http://dx.doi.org/10.1080/02664763.2010.515674.

Wood, J. 2008. "On the Covariance Between Related Horvitz-Thompson Estimators." Journal of Official Statistics 24: 53-78.

Received July 2013

Revised February 2014

Accepted April 2014 